\title{
Effects of Different Imaging Models on Least-Squares Image Reconstruction Accuracy in Photoacoustic Tomography
}

\author{
Jin Zhang, Mark A. Anastasio*, Member, IEEE, Patrick J. La Rivière, Member, IEEE, and \\ Lihong V. Wang, Fellow, IEEE
}

\begin{abstract}
In the classic formulation of photoacoustic tomography (PAT), two distinct descriptions of the imaging model have been employed for developing reconstruction algorithms. We demonstrate that the numerical and statistical properties of unweighted least-squares reconstruction algorithms associated with each imaging model are generally very different. Specifically, some PAT reconstruction algorithms, including many of the iterative algorithms previously explored, do not work directly with the raw measured pressure wavefields, but rather with an integrated data function that is obtained by temporally integrating the photoacoustic wavefield. The integration modifies the statistical distribution of the data, introducing statistical correlations among samples. This change is highly significant for iterative algorithms, many of which explicitly or implicitly seek to minimize a statistical cost function. In this work, we demonstrate that iterative reconstruction by least-squares minimization yields better resolution-noise tradeoffs when working with the raw pressure data than with the integrated data commonly employed. In addition, we demonstrate that the raw-data based approach is less sensitive to certain deterministic errors, such as dc offset errors.
\end{abstract}

Index Terms-Iterative image reconstruction, optoacoustic tomography, photoacoustic tomography, thermoacoustic tomography.

\section{INTRODUCTION}

$\mathbf{P}$ HOTOACOUSTIC tomography (PAT), also known as optoacoustic tomography, is an emerging soft-tissue imaging modality that has great potential for a wide range of biomedical imaging applications. It can be viewed as a hybrid imaging modality in the sense that it utilizes an optical contrast

Manuscript received March 02, 2009; revised May 11, 2009. Current version published October 28, 2009. This work was supported in part by the Pritzker Institute of Biomedical Science and Engineering under Seed Grant \#4-52681 and in part by the National Science Foundation under CAREER Award 0546113. The work of P. J. La Rivière was supported by an American Cancer Society Research Scholar Grant. Asterisk indicates corresponding author.

J. Zhang is with the Radiation Oncology Department, University of Maryland, Baltimore, MD 21201 USA (e-mail: jzhang@umm.edu).

*M. A. Anastasio is with the Department of Biomedical Engineering, Medical Imaging Research Center, Illinois Institute of Technology, Chicago, IL 60616 USA (e-mail: anastasio@iit.edu).

P. J. La Rivière is with the Department of Radiology, The University of Chicago, Chicago, IL 60637 USA (e-mail: pjlarivi@ midway.uchicago.edu).

L. V. Wang is with the Department of Biomedical Engineering, Washington University in St. Louis, St. Louis, MO 63130 USA (e-mail: lhwang @ seas.wustl. edu).

Color versions of one or more of the figures in this paper are available online at http://ieeexplore.ieee.org.

Digital Object Identifier 10.1109/TMI.2009.2024082 mechanism combined with ultrasonic detection principles, thereby combining the advantages of optical and ultrasonic imaging while circumventing their primary limitations. The goal of PAT is to reconstruct the distribution of an object's absorbed optical energy density from measurements of pressure wavefields that are induced via the thermoacoustic effect [1]-[3]. Because the optical absorption characteristics of tissue vary strongly with hemoglobin content, knowledge of the absorbed optical energy distribution can yield both structural and functional information [4]-[7]. Moreover, when employed with targeted probes or optical contrast agents, PAT has the potential to facilitate high-resolution molecular imaging [8], [9] of deep structures, which cannot be achieved easily with pure optical methods. Accordingly, PAT may facilitate a variety of important human and animal imaging studies, and is being developed actively by numerous research groups worldwide.

Photoacoustic tomography is a computed imaging modality and utilizes an image reconstruction algorithm to form an estimate of the absorbed optical energy distribution from knowledge of the measured photoacoustic wavefields. A variety of analytic image reconstruction algorithms have been developed for 3-D PAT assuming canonical measurement apertures [10]-[14]. All known analytic reconstruction algorithms that are mathematically exact and numerically stable require complete knowledge of the photoacoustic wavefield on a measurement aperture that either encloses the entire object or extends to the infinity. In many potential applications of PAT, such as breast imaging, it may not be convenient, or possible, to acquire such measurement data. Because of this, iterative reconstruction algorithms for PAT are being developed actively [15]-[19] that provide the opportunity for accurate image reconstruction from incomplete measurement data. Iterative reconstruction algorithms, such as those based on least squares objective functions, also allow for ready modeling of physical nonidealities in the data such as those introduced by acoustic inhomogeneity and attenuation. Overall, while they also generally demand a larger computational burden than analytic algorithms, iterative reconstruction algorithms offer greater flexibility regarding the choice of measurement aperture, permit utilization of more accurate imaging models, and provide the opportunity for accurate image reconstruction from incomplete measurement data.

Some PAT reconstruction algorithms, including many of the iterative algorithms previously explored, do not work directly with the raw measured pressure wavefields, but rather with an integrated data function, obtained by temporally integrating the 


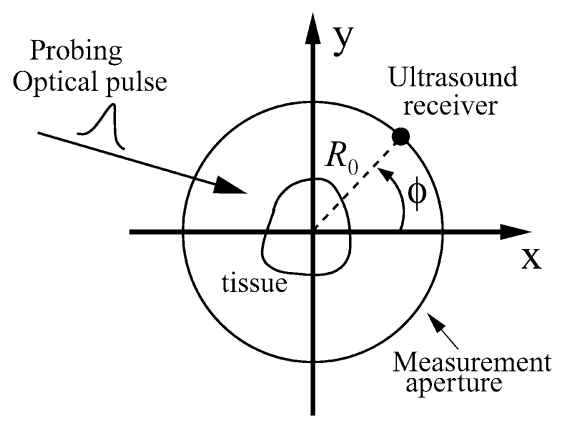

Fig. 1. Imaging geometry of PAT. In the 3-D case, the measurement aperture is a 2 -D surface.

photoacoustic wavefield. For the case of an acoustically homogeneous object, the integrated data function is related to the optical energy distribution via a spherical Radon transform [20], [21]. For ideal data containing no noise or other inconsistencies, this integration step is of little consequence. However, in the presence of noise, the integration modifies the statistical distribution of the data, introducing statistical correlations among samples. This change is highly significant for iterative algorithms, many of which explicitly or implicitly seek to minimize a statistical cost function.

In this work, we demonstrate that iterative reconstruction by unweighted least-squares minimization yields better resolutionnoise tradeoffs when working with the raw pressure data than with the integrated data. In addition, we demonstrate that the raw-data based approach is less sensitive to certain deterministic errors, such as dc offset errors. Finally, we show that the two approaches also have slightly different convergence properties. The concept of data-space preconditioning is employed to analyze and explain these differences.

The remainder of the article is organized as follows. In Section II, the PAT imaging models and iterative reconstruction method employed in our studies are reviewed briefly. We review the concept of data-space preconditioning in Section III, and investigate the numerical properties of least-squares reconstruction algorithms associated with each imaging model. Computer-simulation studies are presented in Section IV, which are corroborated by experimental data studies in Section V. Finally, the article concludes with a discussion and summary of the work in Section VI.

\section{BACKGROUND}

\section{A. PAT Imaging Models in Continuous Forms}

A schematic of the PAT imaging geometry is shown in Fig. 1. A short laser pulse is employed to irradiate an object and subsequently the optical energy is rapidly absorbed. The total energy absorption depends on the optical properties of the tissue, which generally vary with tissue type. For example, a highly vascularized tumor typically absorbs more optical energy than surrounding healthy tissue due to its elevated hemoglobin content. The thermoacoustic effect results in the generation of a pressure wavefield $p(\vec{r}, t)$ [1]-[3], where $\vec{r} \in \mathbb{R}^{3}$ and $t$ is the temporal coordinate. The resulting pressure wavefield can be measured by use of wide-band ultrasonic transducers located on a measurement aperture $\Omega_{0} \subset \mathbb{R}^{3}$, which is a 2-D surface that partially or completely surrounds the object. The coordinate $\vec{r}_{0} \in \Omega_{0}$ will denote a particular transducer location. Assuming the object possesses homogeneous acoustic properties and the duration of the irradiating optical pulse is negligible, the pressure wavefield $p\left(\vec{r}_{0}, t\right)$ at transducer location $\vec{r}_{0}$ can be expressed as [2]

$$
p\left(\vec{r}_{0}, t\right)=\frac{\eta}{4 \pi} \int_{V} d^{3} \vec{r} f(\vec{r}) \frac{d}{d t} \frac{\delta\left(t-\frac{\left|\vec{r}_{0}-\vec{r}\right|}{c_{0}}\right)}{\left|\vec{r}_{0}-\vec{r}\right|}
$$

where the object function $f(\vec{r})$ describes the spatially variant absorbed optical energy density distribution of the object, $\eta$ is the isobaric volume expansion coefficient divided by the specific heat of the medium, $c_{0}$ is the (constant) speed-of-sound in the object and background medium, and $\delta(\cdot)$ denotes the 1-D Dirac delta function. The object function is compactly supported, bounded and nonnegative, and the integration in (1) is performed over its support volume $V$. Equation (1) represents a canonical imaging model for PAT. The reconstruction problem of PAT is to determine a suitable estimate of $f(\vec{r})$ from knowledge of temporally sampled values of the pressure wavefields $p\left(\vec{r}_{0}, t\right)$ recorded at a finite number of locations on $\Omega_{0} \cdot{ }^{1}$

The imaging model in (1) can be recast into a different but mathematically equivalent form. Specifically, by acting $t \int_{0}^{t} d t^{\prime}$ on both sides of (1) one immediately obtains [20]

$$
g\left(\vec{r}_{0}, t\right)=\frac{\eta}{4 \pi} \int_{V} d^{3} \vec{r} f(\vec{r}) \delta\left(t-\frac{\left|\vec{r}_{0}-\vec{r}\right|}{c_{0}}\right)
$$

where the data function $g\left(\vec{r}_{0}, t\right)$ is defined as

$$
g\left(\vec{r}_{0}, t\right) \equiv t \int_{0}^{t} d t^{\prime} p\left(\vec{r}_{0}, t^{\prime}\right)
$$

Note that the integrated data function $g\left(\vec{r}_{0}, t\right)$ represents a scaled version of the acoustic velocity potential [22].

According to (3), knowledge of $p\left(\vec{r}_{0}, t\right)$ is equivalent to knowledge of $g\left(\vec{r}_{0}, t\right)$ (and vice versa), so $g\left(\vec{r}_{0}, t\right)$ can be regarded as a measurable and therefore known quantity. Equation (2) is a spherical Radon transform that relates $g\left(\vec{r}_{0}, t\right)$ to surface integrals of $f(\vec{r})$ over a collection of concentric spheres of radii $c_{0} t$ centered at the transducer location $\vec{r}_{0}$. Accordingly, an estimate of $f(\vec{r})$ can be obtained by use of a reconstruction algorithm that seeks to invert the spherical Radon transform [10], [11], [17], [23].

In the absence of data inconsistencies and finite sampling effects, the estimates of $f(\vec{r})$ obtained by inverting the imaging models in (1) and (2) would be identical. However, in practice when only sampled and noisy values of $p\left(\vec{r}_{0}, t\right)$, and consequently of $g\left(\vec{r}_{0}, t\right)$, are available, the estimates of $f(\vec{r})$ obtained by use of least-squares iterative reconstruction can possess dramatically different statistical properties and artifacts.

\footnotetext{
${ }^{1}$ In practice, the measured $p\left(\vec{r}_{0}, t\right)$ will generally need to be corrected for degradation caused by the temporal and spatial filtering characteristics of the ultrasonic transducer.
} 


\section{B. PAT Imaging Models in Discrete Forms}

In practice, $p\left(\vec{r}_{0}, t\right)$ and $g\left(\vec{r}_{0}, t\right)$ are discretized temporally and measured at a finite number of receiver locations. The vectors $p, g \in Y \equiv \mathbb{R}^{N}$ will represent lexicographically ordered representations of the sampled data functions, where the dimension $N$ is defined by the product of the number of temporal samples acquired at each transducer location and the number of transducer locations. Because we will be focusing on iterative reconstruction algorithms, we will also require a discrete representation of the object function $f(\vec{r})$. The vector $f \in X \equiv \mathbb{R}^{M}$ will denote a lexicographically ordered $M$-dimensional representation of $f(\vec{r})$. Hereafter, the vector spaces $X$ and $Y$ will be referred to as the object- and data-spaces, respectively.

The discrete versions of the imaging models in (1) and (2) can be expressed as

$$
p=P f
$$

and

$$
g=G f
$$

where the operators $P$ and $G$ are $N \times M$ matrix representations of the integral transformation in (1) and the spherical Radon transform in (2), respectively, which map $X \mapsto Y$.

The operators $P$ and $G$ are related by a simple data transformation operator on $Y$

$$
G=C P
$$

where $C$ is an $N \times N$ matrix representation of the weighted integration operator in (3) that maps $Y \mapsto Y$. Alternatively, $P$ and $G$ can be related as

$$
P=D G
$$

where $D$ is a $N \times N$ matrix representation of the differentiationlike operator $(d / d t)(\cdot / t)$ that maps $Y \mapsto Y$.

\section{Statistical Considerations}

For the sampled raw pressure data, in the presence of noise, we have the following discrete imaging equations

$$
p=P f+n_{p}
$$

where $n_{p}$ is a vector of additive noise. The principal source of noise in PAT is electronic noise in the transducer, with no correlation from sample to sample, so $n_{p}$ is well modeled as a multivariate Gaussian random vector with a diagonal covariance matrix $\Pi_{p}=\sigma^{2} I$, where $\sigma^{2}$ is the variance in each sample and $I$ represents the identity matrix. A least-squares solution to the image reconstruction problem working with the $p$ data is given by

$$
\widehat{f}_{p}^{L S}=\arg \min _{f}\|p-P f\|^{2}
$$

where the solution must typically be found iteratively due to the large size of the matrices involved.
For the sampled integrated pressure data, in the presence of noise, we have the following discrete imaging equations:

$$
g=G f+n_{g}
$$

where $n_{g}$ is again vector of additive noise. Because the $g$ data is obtained by acting linearly on the measured $p$ data, $n_{g}$ is also a multivariate Gaussian random vector, with the covariance matrix $\Pi_{g}$ no longer diagonal, but given by $\Pi_{g}=C \Pi_{p} C^{T}$. A least-squares solution to the image reconstruction problem working with the $g$ data is given by

$$
\hat{f}_{g}^{L S}=\arg \min _{f}\|g-G f\|^{2} .
$$

As we will see in what follows, the properties of these two solutions are quite different, with the $p$-based solution being generally superior. One way of seeing this is to note that the $p$-based least-squares solution is in fact the maximum-likelihood solution for the statistical model governing $p$, while the $g$-based least-squares solution is not the maximum-likelihood solution for the statistical model governing $g$. The maximum-likelihood solution for the statistical model governing $g$ would correspond to minimizing a weighted least-squares objective function with the weights matrix given by the inverse of $\Pi_{g}$. It is not hard to show that this would produce the same estimate as the $p$-based least-squares solution.

\section{Review of Object-Space Preconditioning Concepts in Iterative Image Reconstruction}

Consider a general discrete imaging equation of the form

$$
y=A f+n_{y}
$$

where $y \in Y, f \in X$, and $A \in \mathbb{R}^{N \times M}$. In PAT, $y$ and $A$ would correspond to $p$ and $P$ or $g$ and $G$, as described by the two imaging models given above. A least-squares (LS) estimate $\hat{f}$ of $f$ can be obtained by iteratively inverting the system's normal equation

$$
H f=b
$$

where $b \equiv A^{T} y, b \in X$, and $H \equiv A^{T} A$ with $A^{T}$ denoting the transpose of $A$. The operator $H: X \mapsto X$ is known as the Hessian matrix of the least-squares problems.

The goal of preconditioning is usually to improve the convergence rate of iterative algorithms [24]-[26] that invert (10) to determine $\hat{f}$, or a modified version of $(10)$ that yields a regularized estimate [27]. To achieve this, (10) is modified by use of an $M \times M$ matrix (preconditioner) as

$$
M H f=M b .
$$

Note that because $M$ acts on the vector space $X$, the preconditioning of the linear system has been performed in the $o b$ ject-space. 
Iterative algorithms that seek to invert (11) generally possesses different numerical properties than those that seek to invert (10). By properly designing the preconditioner $M$ [24], iterative reconstruction algorithms for determining $\hat{f}$ may possess improved convergence rates [28]. Existing preconditioners include the ideal inverse Hessian, diagonal, circulant and other $a d$ hoc matrices [24].

\section{DATA-SPACE PRECONDITIONING IN PAT}

\section{A. Data-Space Preconditioning Concepts}

To simplify our discussion of data-space preconditioning, we consider the LS image reconstruction problem. However, the general concepts below are applicable to the penalized LS (PLS) estimation problem considered subsequently.

A LS estimate of $f$ as given by (8) corresponds to a solution of the normal equation formed from (4)

$$
\underbrace{G^{T} D^{T} D G}_{\equiv H_{p}} f=G^{T} D^{T} p
$$

where we have used (7). Alternatively, a different LS estimate of $f$ can be computed from the $g$ data as given by (9) which corresponds to a solution of the normal equation formed from (5)

$$
\underbrace{G^{T} G}_{\equiv H_{g}} f=G^{T} C p .
$$

The Hessian matrices corresponding to normal equations in (12) and (13) will be denoted as

$$
H_{p} \equiv G^{T} D^{T} D G
$$

and

$$
H_{g} \equiv G^{T} G
$$

Reconstruction algorithms that implement (8) and (9) (or later, regularized versions of them) will be referred to as $p$-recon and g-recon algorithms.

If we consider (12) to be the normal equation of the "standard" imaging model, then (13) can be interpreted as a preconditioned version of it, or vice versa. However, the form of the preconditioning is distinct from the conventional method of preconditioning described by (11). Specifically, (12) and (13) differ by operators that act on the data-space $Y$, whereas the preconditioning operator $M$ in (11) acts on the object-space $X$. Because of this, we refer to (12) and (13), or equivalently, (4) and (5), as a data-space preconditioned pair of imaging equations.

As described below, because the preconditioned Hessian $H_{g}$ is different than the original Hessian $H_{p}, p$-recon and $g$-recon reconstruction algorithms will have different intrinsic numerical properties. Moreover, as reflected by the right-hand sides of (12) and (13), data-space preconditioning also modifies the measured data vector $p$. Consequently, as demonstrated in Section IV, the erroneous components of $p$ will generally be propagated differently by $p$-recon and $g$-recon algorithms, which can result in estimates $\hat{f}_{p}$ and $\hat{f}_{g}$ that have very different artifacts and statistical properties. The ability of data-space preconditioning to strongly influence the physical and statistical characteristics of the reconstructed image, as opposed to simply the convergence rate of the reconstruction algorithm, distinguishes it from commonly employed conventional object-space preconditioning methods.

\section{B. Intrinsic Numerical Properties of Data-Space Preconditioned Systems in PAT}

To gain insights into certain intrinsic numerical properties of $p$-recon and $g$-recon iterative reconstruction algorithms, we numerically investigated the eigen-spectrum of the Hessian matrices for the two imaging models.

In practice, penalized LS (PLS) estimates [27], [29] are typically computed to mitigate the effects of data noise. In this case, a penalty term $\beta\|K f\|^{2}$ is included in the right-hand sides of (8) and (9), where the scalar $\beta$ is a regularization parameter and $K$ is an $M \times M$ matrix that represents a high-pass filtering operation [see (22) and (23)]. The Hessians in (14) and (15) will then take the forms

$$
H_{g}^{r}=G^{T} G+\beta K^{T} K
$$

and

$$
H_{p}^{r}=G^{T} D^{T} D G+\beta K^{T} K
$$

where the superscript $r$ denotes that the Hessians correspond to the normal equations of the regularized problems.

We considered a canonical 2-D PAT imaging geometry corresponding to a circular measurement aperture. We assume the focused transducer receives and integrates only acoustic pressure signals transmitted from the imaging plane, where the 3-D PAT imaging model given by (1) reduces to the 2-D circular mean model. However, the conclusions below are relevant to other measurement apertures and 3-D PAT imaging model, because our data preconditioning takes place only in the time-dimension that is independent of the dimension of the data-space or the measurement aperture geometry. The sampling conditions, geometry parameters, and choice of $K$ were the same as described in Section IV-A. The eigenvalues of $H_{g}^{r}$ and $H_{p}^{r}$ were numerically computed and plotted in Figs. 2 and 3, corresponding to the cases $\beta=0.01$ and $\beta=0.05$.

These plots reveal that the condition numbers of the Hessian matrices, i.e., the ratio between the highest and lowest eigenvalues are similar for both $H_{g}$ and $H_{p}$. This establishes that the two Hessians have similar condition numbers. We also observed that the eigenvalues of $H_{p}$ are distributed similarly to those of $H_{g}$. This suggests that the $p$-recon iterative algorithm will have similar intrinsic numerical properties and hence similar convergence rates as the $g$-recon algorithm. These assertions are validated in Fig. 4, where the two approaches do appear to converge at similar rates once each reaches small normalized mean square errors (NMSE) values, although $g$-recon appears to start closer to the true image from its first iteration.

\section{Propagation of Data Inconsistencies With Data-Space Preconditioning}

Data-space preconditioning can change the way in which data inconsistencies are propagated into the final object estimate. The pressure data $p$ measured in practice can be expressed as

$$
p_{e}=p+e
$$




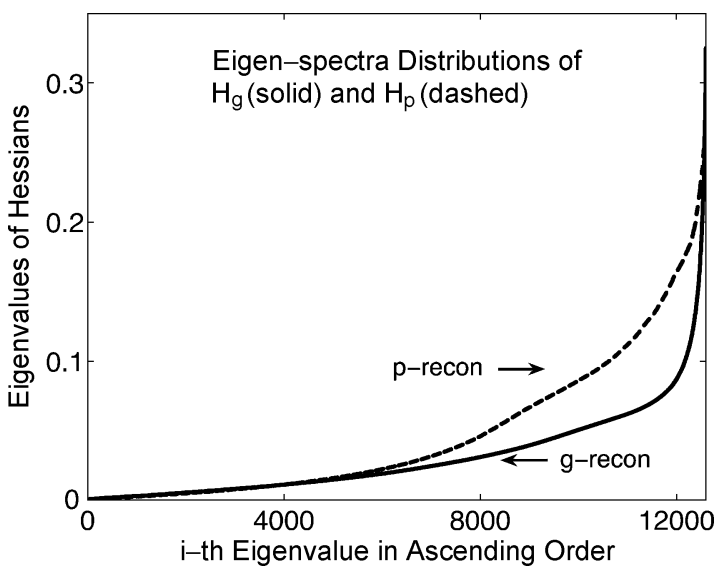

Fig. 2. Eigen-spectra of the Hessian matrices for the two systems, $H_{g}$ (solid) and $H_{p}$ (dashed), corresponding the regularization parameter $\beta=0.01$.

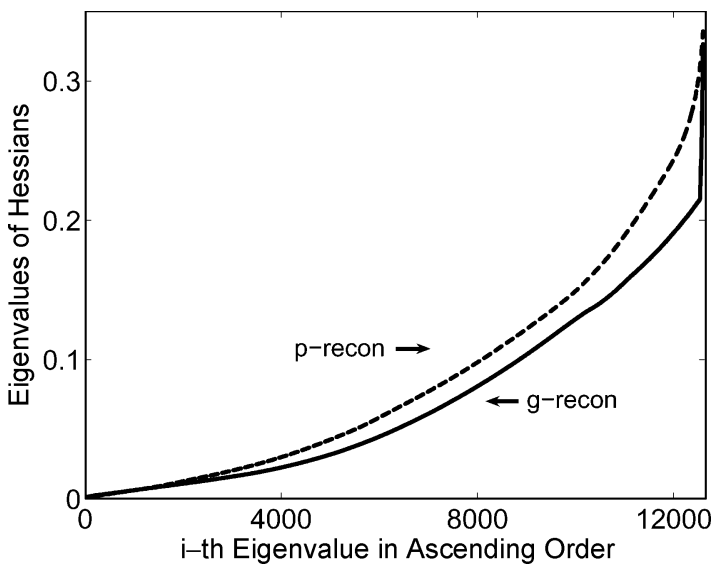

Fig. 3. Eigen-spectra of the Hessian matrices for the two systems, $H_{g}$ (solid) and $H_{p}$ (dashed), corresponding the regularization parameter $\beta=0.05$.

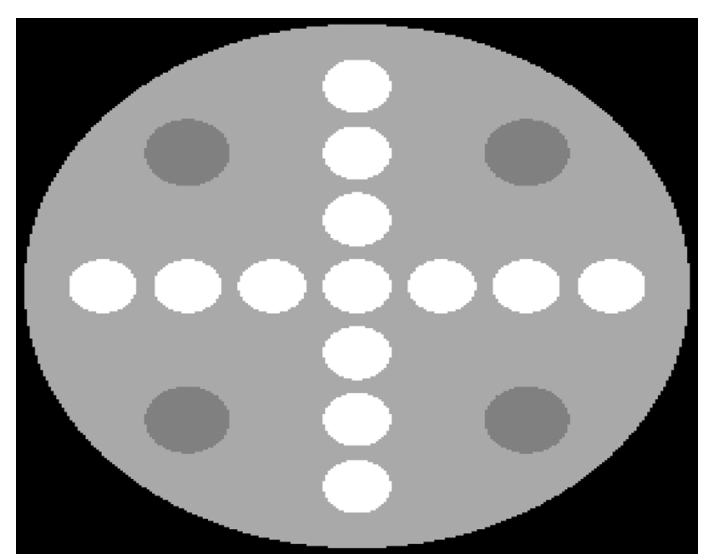

Fig. 4. Two-dimensional numerical phantom representing the object function $f(\vec{r})$.

where the erroneous component $e \in Y$ of the measurement represents stochastic noise as well as deterministic measurement errors caused by medium acoustic heterogeneity, constrained access to measured pressure, finite detector aperture etc. With consideration of this error, the normal equations (for the LS estimation problems) in (12) and (13) take the forms

$$
G^{T} D^{T} D G f=G^{T} D^{T} p+G^{T} D^{T} e
$$

and

$$
G^{T} G f=G^{T} C p+G^{T} C e .
$$

The magnitudes of the terms $G^{T} C e$ and $G^{T} D^{T} e$ determine the extent to which the estimates $\hat{f}_{p}$ and $\hat{f}_{g}$ are perturbed from the corresponding ideal LS estimates.

As a pertinent example of a deterministic data inconsistency, consider the case where the pressure data $p$ recorded at each measurement location are contaminated only by unknown constant (i.e., dc) offsets. This would occur, for example, if the ultrasound transducers employed in the PAT imager were not calibrated precisely but other measurement noises were negligible. The magnitudes of the offsets for different transducers are generally distinct and are described by the components of $e$. Because the integration operator $C$ accumulates the errors but the differentiation operator $D^{T}$ eliminates all dc offsets, we have

$$
\left|G^{T} C e\right|>\left|G^{T} D^{T} e\right|=0 .
$$

Therefore, as demonstrated in Section IV, when dc offsets represent the predominant data inconsistency component, a $p$-recon reconstruction algorithm that inverts (19) can produce an image estimate possessing less severe artifacts and distortions than produced by a $g$-recon reconstruction algorithm that inverts (20).

\section{NUMERICAL INVESTIGATIONS OF RECONSTRUCTION ALGORITHMS}

Computer-simulation studies were conducted to investigate and compare iterative $g$-recon and $p$-recon algorithms. Studies that utilize experimental data are described subsequently in Section V.

\section{A. Simulation Data and Reconstruction Algorithms}

Phantom and Simulation Data: The 2-D numerical phantom shown in Fig. 4 was taken to represent the object function $f(\vec{r})$. It was comprised of uniform disks possessing different gray levels, radii, and locations. The radius of the phantom was 1.0 (arbitrary units). Although we considered the 2-D problem for computational convenience, our conclusions will be generally applicable to 3-D PAT. A circular measurement aperture of radius 1.2 that enclosed the object was employed. At each of 240 uniformly spaced transducer locations $\vec{r}_{0}$ on the measurement circle, simulated pressure data $p\left(\vec{r}_{0}, t\right)$ were analytically computed according to (1), from knowledge of $f(\vec{r})$. For simplicity we did not consider the band-pass characteristics of transducer used in practice, however the conclusions from these simulation studies and their theoretical values are not affected by this fact. At each transducer location 300 temporal samples of $p\left(\vec{r}_{0}, t\right)$ were computed. Accordingly, the pressure vector $p \in Y$ was a column vector of length $240 \times 300$. The noiseless integrated data vector $g=C p$ was computed by use of a discrete approximation of (3).

Erroneous versions of the data sets were computed by including stochastic or deterministic data errors. To model stochastic measurement errors, the pressure data $p$ was treated as an uncorrelated zero-mean Gaussian random vector with different variance levels $\sigma^{2}$ as described below. Noisy versions of 
$p$ were computed as realizations of this stochastic process. From noisy versions of $p$, noisy data vectors $g$ were computed. The effects of integration on the statistical properties of pressure data were discussed in more detail in [15]. Additional sets of pressure data $p$ were generated that contained deterministic offsets $e\left(\vec{r}_{0}\right)$, as would be produced by transducer dc shifts, whose magnitudes are described below. From these versions of $p$, the corresponding data vectors $g$ were computed.

Reconstruction Algorithms: From the simulated $p$ and $g$ data vectors, reconstruction algorithms were implemented for determining object estimates $\hat{f}$. A regularized iterative $p$-recon reconstruction algorithm was implemented that determined an object estimate $\hat{f}_{p}$ as

$$
\hat{f}_{p}=\underset{f}{\operatorname{argmin}}\|p-P f\|^{2}+\beta\|K f\|^{2}
$$

where $K$ is a discrete implementation of the differential operator $(\partial / \partial x)+(\partial / \partial y)$. Similarly, a regularized iterative $g$-recon reconstruction algorithm was implemented that determined an object estimate $\hat{f}_{g}$ as

$$
\hat{f}_{g}=\underset{f}{\operatorname{argmin}}\|g-G f\|^{2}+\beta\|K f\|^{2} .
$$

The object $f(\vec{r})$ was described by a $256 \times 256$ matrix, corresponding to a standard 2-D pixel basis representation, so the lexicographically ordered $f \in X$ was of length $256^{2}$. The discrete circular Radon transform $G$ was implemented by numerical integration, similar to the ray-driven technique used to simulate X-ray sinograms [30], specifically by dividing the corresponding integration arc into equally spaced segments and then summing up the values of the object function on each segment. Providing the segment length (integration step) is small and data samples are dense compared with the pixel size, this numerical integration closely represents the corresponding analytical circular Radon transform. A discrete form of operator $P$ was implemented by performing a simple finite difference after numerical integration operator $G$. Equations (22) and (23) were solved by use of the Fletcher-Reeves version of conjugate gradient (CG) algorithm [31] for different values of the regularization parameter $\beta$. The $\mathrm{CG}$ algorithms were terminated after 60 iterations, which ensured convergence as described below.

\section{B. Results from Consistent Data}

Object estimates $\hat{f}_{g}$ and $\hat{f}_{p}$ reconstructed from consistent (perfect) data are shown in Fig. 5(a) and (b), and vertical profiles through the centers of the images are superimposed in Fig. 5(c). As expected, both estimates are very similar and accurately represent the true object $f$. To compare the convergence rates of the $g$-recon and $p$-recon algorithms, we examined NMSEs of the reconstructed images versus iteration number of the $\mathrm{CG}$ algorithm. The NMSE is defined as $\left\|f-f_{k}\right\| /\|f\|$, where $f_{k}$ is the estimate in the $k$ th iteration and $f$ is true object. For the case of $\beta=0.01$, shown in Fig. 6 , the $p$-recon algorithm started off with a relatively higher NMSE but also initially converged at a faster rate than the $g$-recon algorithm. After approximately 30 iterations both algorithms had approximately converged and produced small NMSEs that did not change significantly with additional iterations. In the studies below, we terminated the

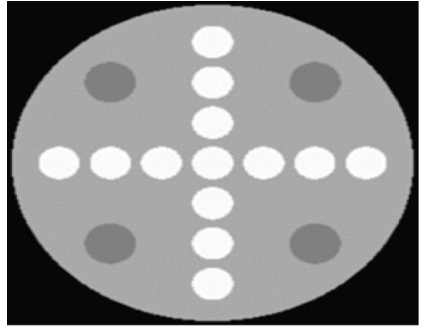

(a)

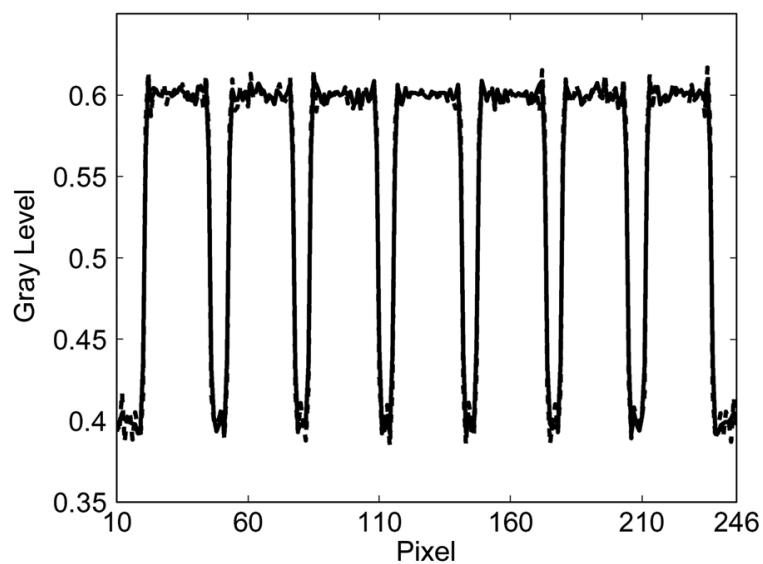

(c)

Fig. 5. Images reconstructed from consistent (noise-free) data by use of the (a) $g$-recon and (b) $p$-recon algorithms. (c) Vertical profiles through the center of subfigure (a) (solid line) and subfigure (b) (dashed line).

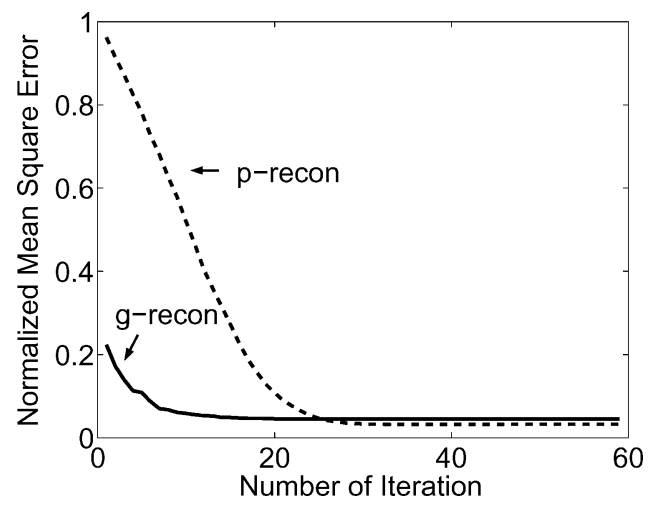

Fig. 6. NMSE versus iteration number for the $g$-recon (solid line) and $p$-recon (dashed line) algorithms applied to consistent data.

$\mathrm{CG}$ algorithms at 60 iterations, which ensured convergence when the data were erroneous and/or other values of $\beta$ were employed.

\section{Results From Data Containing Random Inconsistencies}

From ensembles of $p$ and $g$ containing 400 noisy realizations, ensembles of noisy images were reconstructed by use of the $g$-recon and $p$-recon algorithms, corresponding to different choices of the regularization parameter $\beta$. From each collection of images, empirical estimates of the image standard deviation (std) were computed within a $16 \times 16$ pixel region in the center of reconstructed images corresponding to both reconstruction algorithms. We quantified the resolution of reconstructed images by fitting the rising edge of a pre-chosen structure (we 


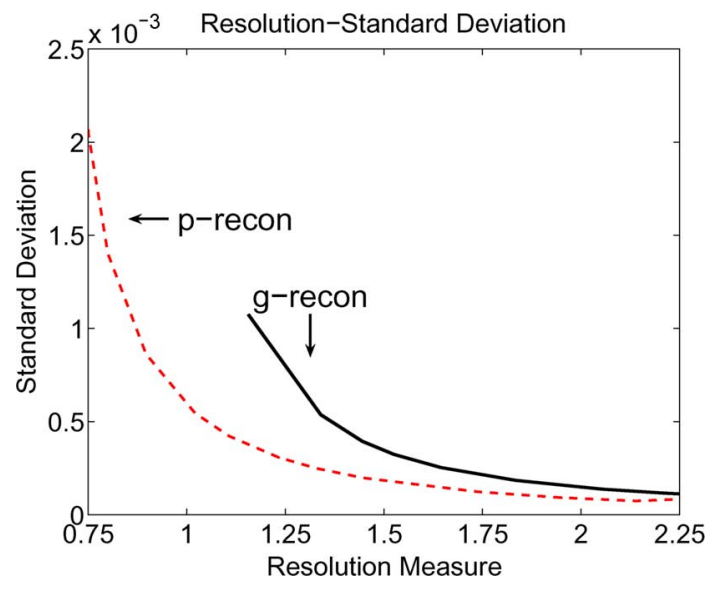

Fig. 7. Resolution-standard deviation curves corresponding to the $g$-recon (solid line) and $p$-recon (dashed line) algorithms.

chose the central disk in this study) in the empirical mean images to an error function [32]. The image resolution was characterized by the full-width at half-maximum (FWHM) value of the fitted error function [32].

Resolution versus standard deviation curves were constructed by plotting the (resolution, std) pairs obtained for different values of the regularization parameter $\beta$ ranging from 0.0 to 0.5 . The curves for the $p$-recon and $g$-recon algorithms are shown in Fig. 7, for the case where the data $p$ were contaminated by noise with level $\sigma=0.01$. This noise level approximately corresponds to $3 \%$ of the peak value of the noiseless $p$ data. In order to display the two resolution-noise curves in their common $y$-axis range, we truncated the $p$-recon curve at FWHM $=0.75$. When $\beta=0$, which corresponds to the upper left-most region of the figures, the $p$-recon algorithm can achieve higher resolutions than the $g$-recon algorithm. In their common resolution region, the resolution-std curve corresponding to the $p$-recon algorithm is everywhere lower than the curve corresponding to the $g$-recon algorithm, indicating that the $p$-recon algorithm will generally produce images with lower noise levels at matched resolution as $g$-recon.

Figs. 8 and 9 contain images reconstructed from data containing noise levels of $\sigma=0.005$ and $\sigma=0.05$, which were approximately $1.5 \%$ and $15 \%$ of the peak signal strength, respectively. In both reconstructions, the parameters $\beta$ in front of the penalty term are chosen at matched resolution on the resolution-noise curves for the fairness of the comparison: $\beta=0.070$ for $p$-recon and $\beta=0.001$ for $g$-recon, both correspond to resolution measure around FWHM $=1.35$. One can visually verify that the image produced by the $p$-recon algorithm possesses slightly lower noise level than the one produced by the $g$-recon algorithm when the spatial resolutions of the two are comparable.

One can also notice the apparent differences in the noise texture in images reconstructed from $g$-recon and $p$-recon. There are visually obvious "lumpy" textures in the $g$-recon image due to the noise correlation in the $g$ data, which in turn propagates through the reconstructing algorithm and forms the texture. A systematic investigation of how differences in the noise textures affect task-based measures of image quality (e.g., object de-

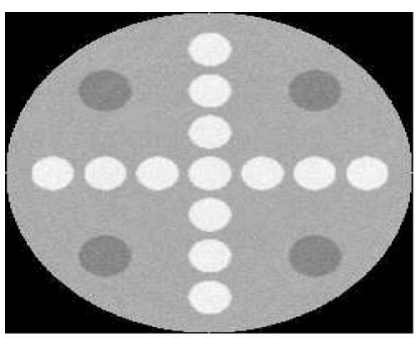

(a)

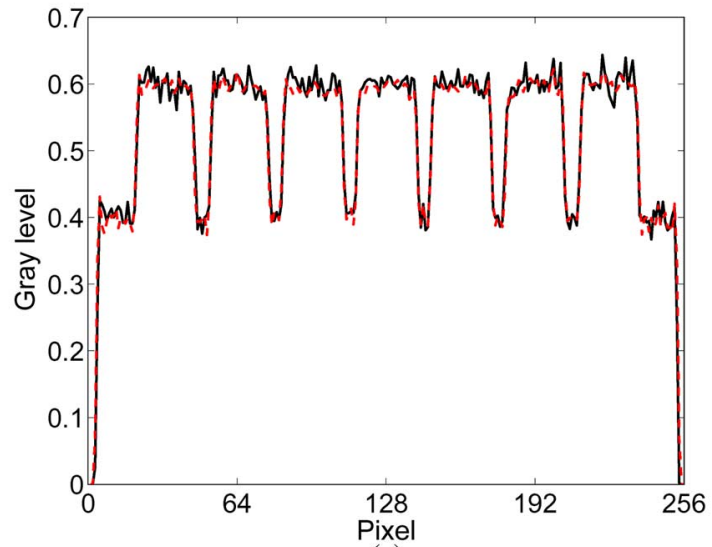

(c)

Fig. 8. Images reconstructed from data containing low levels of Gaussian noise (see text) by use of the (a) $g$-recon and (b) $p$-recon algorithms. (c) Vertical profiles through the center of subfigure (a) (solid line) and subfigure (b) (dashed line).

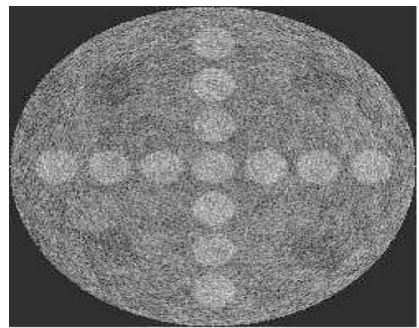

(a)

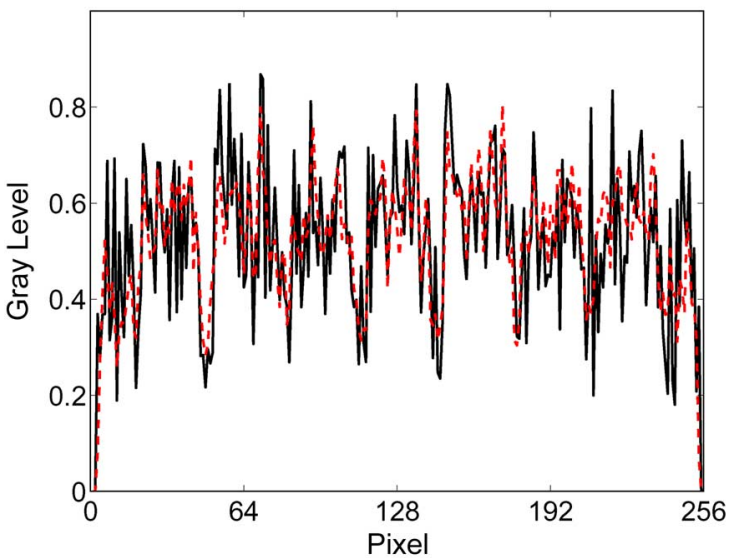

(c)

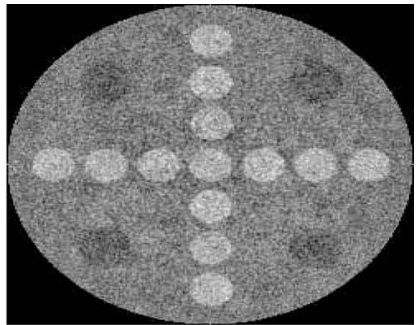

(b)
Fig. 9. Images reconstructed from data containing high levels of Gaussian noise (see text) by use of the (a) $g$-recon and (b) $p$-recon algorithms. (c) Vertical profiles through the center of subfigure (a) (solid line) and subfigure (b) (dashed line).

tectability studies) is beyond the scope of this article, but in general highly correlated noise tends to degrade signal detectability. 


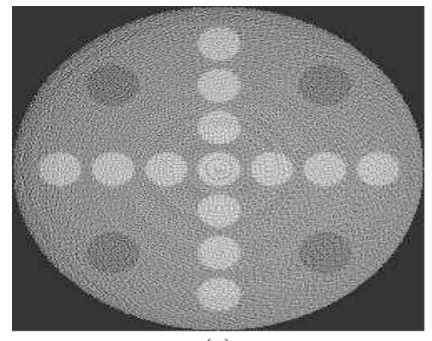

(a)

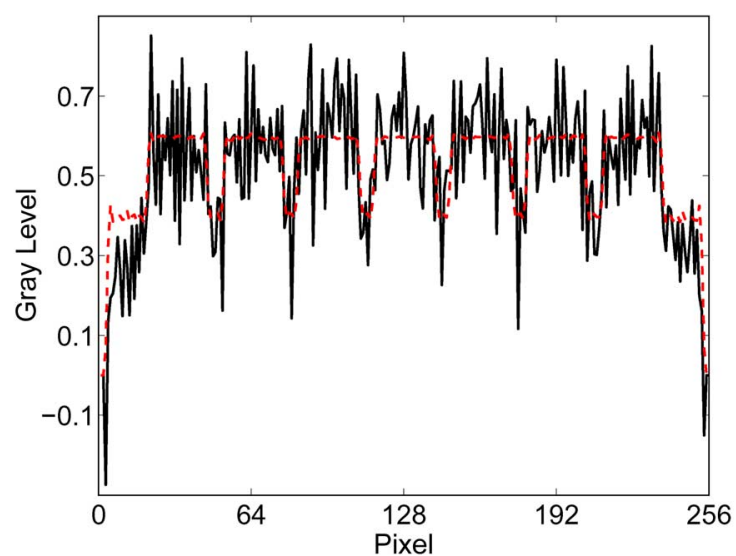

(c)

Fig. 10. Images reconstructed from data containing deterministic DC offsets (see text) by use of the (a) $g$-recon and (b) $p$-recon algorithms. (c) Vertical profiles through the center of subfigure (a) (solid line) and subfigure (b) (dashed line).

\section{Studies Employing Data Containing Deterministic Inconsistencies}

Additional data sets were generated that contained deterministic offsets in the pressure data $p$. For the case where the magnitudes of the offsets were $e=0.0125$, which corresponded to approximately $4 \%$ of the peak signal strength, images reconstructed by use of the $g$-recon and $p$-recon algorithms are displayed in Fig. 10(a) and (b). Profiles through the reconstructed images are shown in Fig. 10(c). As predicted by our analysis, when the data contain spatially uniform errors, e.g., as would be produced by miscalibrated transducers, the image reconstructed by use of the $g$-recon algorithm [Fig. 10(a)] contains severe distortions and ringing artifacts while the image obtained by use of the $p$-recon algorithm [Fig. 10(b)] is not strongly affected by the errors and closely resembles the true phantom. This is because the dc errors are accumulated by the integration operation in the $g$-recon algorithm, which introduces large errors into the input data, while the error is annihilated by the differentiation operator in the $p$-recon algorithm. It is worth noting that because of the band-pass-filtering effect of some transducers, experimental data in certain implementations may not contain such large dc errors as considered in this simulation study.

\section{EXPERIMENTAL RESULTS}

Images were reconstructed from experimental data sets acquired previously in the Optical Imaging Laboratory at Wash-

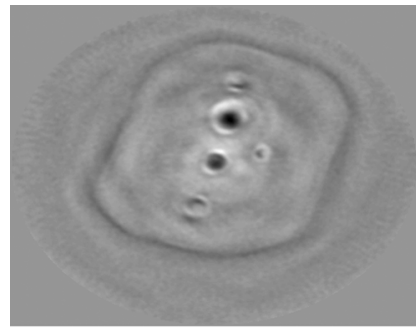

(a)

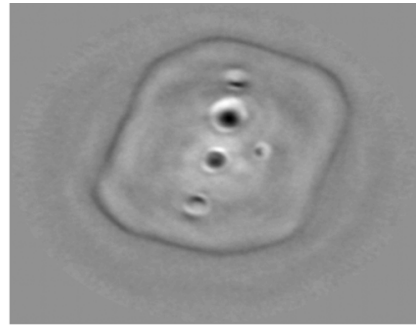

(b)
Fig. 11. Images reconstructed from experimental data corresponding to a physical phantom by use of the (a) $g$-recon and (b) $p$-recon algorithms.

ington University in St. Louis. ${ }^{2}$ The reconstructed image size was $400 \times 400$ pixels, and the regularization parameters $\beta$ in the $\mathrm{CG}$ algorithms were manually chosen to yield reconstructions whose visual appearances were deemed to be closest to the true object. The grey scale window of the reconstructed images was fixed at $[-0.1,0.1]$.

The first data set corresponded to a tissue phantom that is described in [33]. The data were acquired using a microwave source instead of a laser and a 2-D circular measurement geometry that had a scanning radius $R_{0}=70 \mathrm{~mm}$ [33]. Pressure signals were measured at 160 equally spaced positions on the measurement circle, and each signal contained 2000 temporal samples acquired at a $50 \mathrm{MHz}$ sampling rate. The images in Figs. 11(a) and (b) were reconstructed by use of the $g$-recon and $p$-recon algorithms. The image reconstructed by use of the $p$-recon algorithm [Fig. 11(b)] possesses better visual image quality, specifically higher spatial resolution. We verified that the image reconstructed by use of the $g$-recon algorithm [Fig. 11(a)] could not achieve comparable visual quality by use of alternative $\beta$ values.

The second data set corresponded to a photoacoustic tomography imaging study of a mouse brain. As described in [34], the data were acquired using an laser source and a 2D circular measurement geometry that had a scanning radius $R_{0}=19.95 \mathrm{~mm}$. Measurements were taken at 240 equally spaced positions on the scanning aperture and for each measurement position, 5000 pressure samples were received at a $100 \mathrm{MHz}$ sampling rate. The image reconstructed by use of the $p$-recon algorithm [Fig. 12(b)] possesses sharper visual image resolution, with comparable noise level as the image reconstructed by use of the $g$-recon algorithm [Fig. 12(a)]. We verified that the visual image quality of $p$-recon result could not be matched by use of any alternative $\beta$ values of $g$-recon.

A third data set corresponded to a thermoacoustic tomography imaging study of a monkey brain [35]. As described in [35], three needles were inserted in the brain. The measurement geometry and number of transducer locations were the same as described above for the first data set. At each transducer location, the pressure signal was sampled at a $20 \mathrm{MHz}$ rate. The image reconstructed by use of the $g$-recon algorithm [Fig. 13(a)] possesses better visual image quality, specifically lower noise levels. We verified that the image reconstructed by use of the

2Department of Biomedical Engineering, Washington University at St. Louis, Director: Prof. L. V. Wang, http://oilab.seas.wustl.edu. 


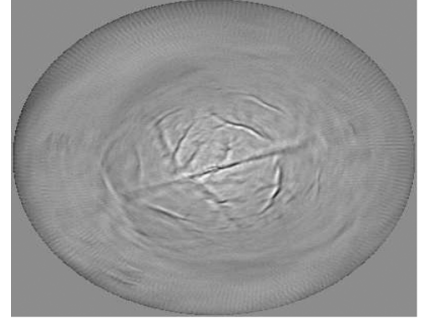

(a)

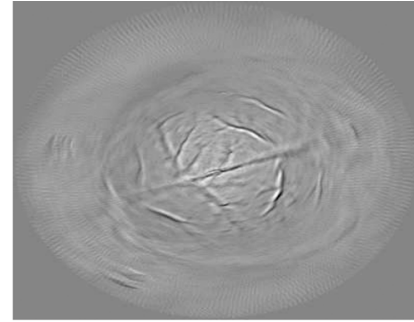

(b)
Fig. 12. Images reconstructed from experimental data corresponding to a mouse brain by use of the (a) $g$-recon and (b) $p$-recon algorithms.

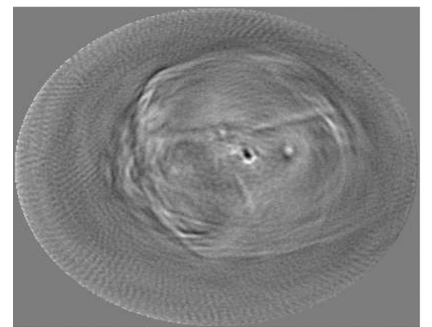

(a)

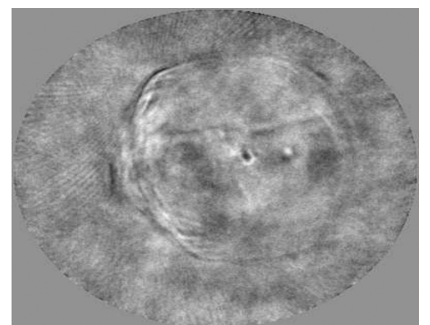

(b)
Fig. 13. Images reconstructed from experimental data corresponding to a primate brain by use of the (a) $g$-recon and (b) $p$-recon algorithms.

$p$-recon algorithm [Fig. 13(b)] could not achieve comparable visual quality by use of alternative $\beta$ values. Due to acoustic heterogeneities, the measured data in this case are expected to be highly inconsistent with respect to the assumed spherical Radon transform imaging model. This example demonstrates that when the data inconsistencies are not described by dc offsets or uncorrelated noise, the $g$-recon algorithm may produce more favorable image estimates than the $p$-recon algorithm in certain applications.

\section{SUMMARY}

Two distinct descriptions of the classic PAT imaging model have been employed for developing reconstruction algorithms. Both imaging models embody the same imaging physics and are mathematically identical. However, the numerical and statistical properties of reconstruction algorithms associated with each imaging model are generally very different, and have not been investigated previously.

Certain PAT reconstruction algorithms, including many of the iterative algorithms previously explored, do not work directly with the raw measured pressure wavefields, but rather with an integrated data function, obtained by temporally integrating the photoacoustic wavefield. The integration modifies the statistical distribution of the data, introducing statistical correlations among samples. This change is highly significant for iterative algorithms, many of which explicitly or implicitly seek to minimize a statistical cost function.

In this paper, we have demonstrated that iterative reconstruction by least-squares minimization yields better resolution-noise tradeoffs when working with the raw pressure data than with the integrated data commonly employed. In addition, we demonstrated that the raw-data based approach is less sensitive to certain deterministic errors, such as dc offset errors.
It is worth noting that the different results obtained when using these two different imaging models is not an inherent property of the models but rather the result of their interaction with the unweighted least-squares objective function, which is not invariant to general linear transformations of the input data. As was mentioned, it can be shown that an objective function based on the use of a weighted least-squares data-agreement term, with weighting matrix proportional to the inverse of the data covariance matrix, is invariant to such transformations and is the maximum-likelihood estimator for the Gaussian noise models assumed here.

Although not investigated in this work, conventional objectspace preconditioning methods [24] can be employed with either of the PAT imaging models and accelerated versions of the $p$-recon and $g$-recon algorithms can be obtained. Because the object-space preconditioning methods do not directly modify the form of data inconsistencies, they typically will not change the physical characteristics of the reconstructed image in a significant way. This data-space preconditioning concept can be readily generalized to other imaging modalities such as X-ray CT and to other iterative algorithms [36]. The investigation of alternative data-space preconditioning transformations for PAT and other tomographic imaging modalities that can favorably influence the physical and/or statistical properties of the reconstructed image remains an interesting topic for future research.

\section{ACKNOWLEDGMENT}

The authors would like to thank the anonymous reviewers for insightful reviews and especially Associate Editor J. Fessler, whose healthy skepticism helped us uncover a bug that had led us to an erroneous conclusion in an earlier version of the manuscript. J. Zhang thanks Dr. M. Jin for helpful discussions.

\section{REFERENCES}

[1] A. A. Oraevsky and A. A. Karabutov, "Optoacoustic tomography," in Biomedical Photonics Handbook, T. Vo-Dinh, Ed. Boca Raton, FL: CRC, 2003.

[2] M. Xu and L. V. Wang, "Biomedical photoacoustics," Rev. Sci. Instrum., vol. 77, no. 041101, 2006.

[3] A. C. Tam, "Application of photo-acoustic sensing techniques," Rev. Mod. Phys., vol. 58, pp. 381-431, 1986.

[4] R. Esenaliev, A. A. Karabutov, and A. A. Oraevsky, "Sensitivity of laser opto-acoustic imaging in detection of small deeply embedded tumors," IEEE J. Sel. Top. Quant., vol. 5, pp. 981-988, Jul./Aug. 1999.

[5] R. Zemp, R. Bitton, M. Li, K. K. Shung, G. Stoica, and L. V. Wang, "Photoacoustic imaging of the microvasculature with a high-frequency ultrasound array transducer," J. Biomed. Opt., vol. 12, p. 010501, 2007.

[6] X. Wang, Y. Pang, G. Ku, X. Xie, G. Stoica, and L. V. Wang, "Noninvasive laser-induced photo-acoustic tomography of the brain in vivo: Structural and functional neuroimaging," Nature Biotechnol., vol. 21, p. 803, 2003.

[7] L. Li, R. Zemp, G. Lungu, G. Stoica, and L. V. Wang, "Photoacoustic imaging of gene lacz expression in living mice," J. Biomed. Opt., vol. 12, p. 020504, 2007.

[8] Y. Wang, X. Xie, X. Wang, G. Ku, K. L. Gill, D. P. ONeal, G. Stoica, and L. V. Wang, "Photoacoustic tomography of a nanoshell contrast agent in the in vivo rat brain," Nano Lett., vol. 4, pp. 1689-1692, 2004.

[9] R. A. Kruger, W. L. Kiser, D. R. Reinecke, G. A. Kruger, and K. D. Miller, "Thermoacoustic optical molecular imaging of small animals," Molec. Imag., vol. 2, pp. 113-123, 2003.

[10] L. A. Kunyansky, "Explicit inversion formulae for the spherical mean radon transform," Inv. Probl., vol. 23, pp. 373-383, 2007.

[11] D. Finch, M. Haltmeier, and Rakesh, "Inversion of spherical means and the wave equation in even dimensions," SIAM J. Appl. Math., vol. 68, no. 2, pp. 392-412, 2007. 
[12] Y. Xu and L. V. Wang, "Universal back-projection algorithm for photoacoustic computed tomography," Phys. Rev. E, vol. 71, no. 016706, 2005.

[13] D. Finch, S. Patch, and Rakesh, "Determining a function from its mean values over a family of spheres," SIAM J. Math. Anal., vol. 35, pp. 1213-1240, 2004

[14] Y. Xu, D. Feng, and L. V. Wang, "Exact frequency-domain reconstruction for thermoacoustic tomography: I. Planar geometry," IEEE Trans. Med. Imag., vol. 21, no. 7, pp. 823-828, Jul. 2002.

[15] M. A. Anastasio, J. Zhang, X. Pan, Y. Zou, G. Keng, and L. V. Wang, "Half-time image reconstruction in thermoacoustic tomography," IEEE Trans. Med. Imag., vol. 24, no. 2, pp. 199-210, Feb. 2005.

[16] M. A. Anastasio, J. Zhang, E. Y. Sidky, Y. Zou, D. Xia, and X. Pan, "Feasibility of half-data image reconstruction in 3D reflectivity tomography with a spherical aperture," IEEE Trans. Med. Imag., vol. 24, no. 2, pp. 1100-1112, Feb. 2005.

[17] X. Pan, Y. Zou, and M. A. Anastasio, "Data redundancy and reducedscan reconstruction in reflectivity tomography," IEEE Trans. Image Process., vol. 12, no. 7, pp. 784-795, Jul. 2003.

[18] P. Ephrat, L. Keenliside, A. Seabrook, F. S. Prato, and J. J. L. Carson, "Three-dimensional photoacoustic imaging by sparse-array detection and iterative image reconstruction," J. Biomed. Opt. vol. 13, no. 5, p. 054052, 2008 [Online]. Available: http://link.aip.org/link/?JBO/13/ $054052 / 1$

[19] G. Paltauf, J. Viator, S. Prahl, and S. Jacques, "Iterative reconstruction algorithm for optoacoustic imaging," J. Acoust. Soc. Amer., vol. 112, pp. 1536-1544, 2002.

[20] R. A. Kruger, P. Liu, R. Fang, and C. Appledorn, "Photoacoustic ultrasound (PAUS) reconstruction tomography," Med. Phys., vol. 22, pp. $1605-1609,1995$

[21] F. Natterer, The Mathematics of Computerized Tomography. New York: Wiley, 1986.

[22] P. M. Morse and K. U. Ingard, Theoretical Acoustics. Princeton, NJ: Princeton Univ. Press, 1986.

[23] S. Norton and M. Linzer, "Ultrasonic reflectivity imaging in three dimensions: Exact inverse scattering solutions for plane, cylindrical, and spherical apertures," IEEE Trans. Biomed. Eng., vol. 28, no. 2, pp. 202-220, Feb. 1981.
[24] J. A. Fessler and S. D. Booth, "Conjugate-gradient preconditioning methods for shift-variant PET image reconstruction," IEEE Trans. Image Process., vol. 8, no. 5, pp. 688-699, May 1999.

[25] N. H. Clinthorne, T. Pan, P.-C. Chiao, W. L. Rogers, and J. A. Stamos, "Preconditioning methods for improved convergence rate in iterative reconstructions," IEEE Trans. Med. Imag., vol. 12, no. 1, pp. 78-83, Mar. 1993.

[26] G. Chinn and S. Huang, "A fast preconditioned conjugate gradient algorithm for regularized WLS reconstruction for PET," in Nucl. Sci. Symp. Med. Imag. Conf. Rec., 1995.

[27] J. A. Fessler, "Penalized weighted least-squares reconstruction for positron emission tomography," IEEE Trans. Med. Imag., vol. 13, no. 2, pp. 290-300, 1994.

[28] G. H. Golub and C. F. V. Loan, Matrix Computations. Baltimore, MD: John Hopkins Univ. Press, 1996.

[29] M. Bertero and P. Boccacci, Inverse Problems in Imaging. Bristol, U.K.: Inst. Physics, 1998.

[30] A. C. Kak and M. Slaney, Principles of Computerized Tomographic Imaging. New York: IEEE Press, 1988.

[31] S. A. Teukolsky, W. T. Vetterling, and B. P. Flannery, Numerical Recepies in C. Cambridge, U.K.: WH Press, 1988.

[32] P. J. La Rivière, "Penalized-likelihood sinogram smoothing for lowdose CT," Med. Phys., vol. 32, pp. 1676-1683, 2005.

[33] M. Xu and L. V. Wang, "Time-domain reconstruction for thermoacoustic tomography in a spherical geometry," IEEE Trans. Med. Imag., vol. 21, no. 7, pp. 814-822, Jul. 2002.

[34] G. Ku, X. Wang, G. Stoica, and L. V. Wang, "Multiple-bandwidth photoacoustic tomography," Phys. Med. Biol., vol. 49, pp. 1329-1338, 2004.

[35] Y. Xu and L. V. Wang, "Rhesus monkey imaging through intact skull with thermoacoustic tomography," IEEE Trans. Ultrason. Ferroelect., vol. 53, no. 3, pp. 542-548, Mar. 2006.

[36] J. Zhang, D. Shi, M. A. Anastasio, J. Sillanpaa, and J. Chang, "Weighted expectation maximization reconstruction algorithms with application to gated megavoltage tomography," Phys. Med. Biol., vol. 50, pp. 299-307, 2005. 